\title{
NOTES ON NEW WORLD ZINGIBERACEAE II
}

\author{
P. J.M.MAAS
}

Instituut voor Systematische Plantkunde, Utrecht A new species from Venezuela has to be added to the genus Renealmia recently monographed by the
author for Flora Neotropica.

Renealmia choroniensis Maas spec. nov.

Renealmia species B, Maas, Fl. Neotropica 18: 145. 1977.

Herba $0.7-2.0 \mathrm{~m}$ alta. Vagina striata. Ligula ca $1 \mathrm{~mm}$ longa. Petiolus ad $30 \mathrm{~mm}$ longus. Lamina anguste elliptica, base longe attenuata, apice longe acuminata, 23-31 cm longa, 4.5-7 cm lata, 6-9-plicata, glabra. Scapus erectus ad prostratus, 4-20 cm longus. Inflorescentia basalis, racemosa, saepe secunda, 3-15 cm longa, $1.5-5 \mathrm{~cm}$ lata. Bracteae roseo-rubrae, mox brunneae decolorantes, persistentes, ovatae, obtusae, $10-25 \mathrm{~mm}$ longae, $10-16 \mathrm{~mm}$ latae. Pedunculi 2-10 $\mathrm{mm}$ longi, pedicelli 2-3 mm longi. Bracteolae roseo-rubrae, 13-22 mm longae.Calyx roseoruber, tubulosus, 13-19 mm longus. Corolla lutea, 27-28 mm longa. Labellum luteum, 9-10 mm longum. Anthera $8 \mathrm{~mm}$ longa. Capsula roseo-viridis, maturitate purpurascens, ellipsoidea ad globosa, 15-20 mm longa, 25-30-seminalis.

TYPE. Steyermark \& Huber 114227 (holotype, U; isotype, VEN), moist, steep, rocky slopes near Regresiva del Diablo, Parque Nacional 'Henry Pittier', alt. $1500 \mathrm{~m}$, edo. Aragua, Venezuela.

Venezuela, Aragua: Parque Nacional 'Henry Pittier', cloud forest near Regresiva del Diablo, alt. 1500-1550 m, Foldats 3889 (VEN), Maas \& Badillo 2171 (MY), Steyermark \& Huber 114257 (U, VEN), 114259,114260 (VEN).

This species has its closest relative in R. alpinia.

In my revision of Renealmia I provisionally indicated this species as 'species B', because the material which was then available for study was incomplete. Soon after my publication Dr. Julian A. Steyermark kindly provided me with some very rich additional collections. This enabled me to complete the description. 